\title{
Involvement of intestinal hypoperfusion related with Indoleamine 2,3-peroxidase activation and 5-HT metabolism abnormality in intestinal dysfunction after acute myocardial infarction
}

xiaofang lu

Beijing Hospital of Traditional Chinese Medicine Affiliated to Capital Medicine University

PINGPING ZHOU

He Bei Colloge of traditional Chinese Medicine

YANGANG WANG ( $\square$ piwei001@163.com )

https://orcid.org/0000-0003-1298-9963

YUEFEN WANG

Beijing Hospital of Traditional Chinese Medicine Affiliated to Capital Medical University

Research

Keywords: Acute myocardial infarction, Intestinal dysfunction, Intestinal hypo-perfusion, Indoleamine 2,3peroxidase, 5-hydroxytryptamine

Posted Date: May 21st, 2020

DOI: https://doi.org/10.21203/rs.3.rs-29062/v1

License: (9) This work is licensed under a Creative Commons Attribution 4.0 International License.

Read Full License 


\section{Abstract \\ Background}

To investigate the intestinal dysfunction after acute myocardial infarction (AMI) and discuss the underlying mechanism.

\section{Methods}

Rats were divided into three groups randomly, including AMI group, Sham group and Normal (N) group. An AMI model was established with ligating the left anterior descending artery (LAD) without ventilator assisted. The body surface electrocardiogram (ECG), HE staining of myocardial tissues and echocardiogram were used to evaluate whether the model was established successfully. The HE staining for ileum tissue was applied to evaluate the structure of the ileum, the intestinal propulsive rate were conducted to investigate the intestinal dysfunction, and laser speckle technique was developed in order to measure the mesenteric blood flow. immunohistochemical method was used to determined the expression of Indoleamine 2, 3-dioxygenase (IDO) in ileum, high-performance liquid chromatography was used to detected 5-hydroxytryptamine(5-HT) metabolism in rats plasma and ileum.

\section{Results}

After AMI in rats, the ECG shows ST segment elevation in lead $\nabla$ for more than 30 minutes and pathological Q wave appeared at 4 weeks after surgery. HE staining showed at 4 weeks after AMI, the ventricular wall of the infarcted area of the rats became thin and white. Echocardiogram showed Left ventricular internal diameters of systole(LVIDs) and Left ventricular internal diameters of diastole(LVIDd) in the AMI group increased significantly, and Interventricular septal thickness at end diastole(IVSd) decreased significantly. Left ventricular ejection fraction(LVEF) and Fractional shortening(FS) values in the AMI group were significantly decreased. HE staining showed intestinal mucosa was hyperemia, edema, and it was infiltrated by a large number of neutrophils. The intestinal propulsive rate was increased in AMI group. Laser speckle technique shows the mesenteric blood flow was decreased in AMI group. Immunohistochemistry showed the expression of IDO was increased in AMI group. Highperformance liquid chromatography showed the 5-HT content in the plasma was increased, and the content of 5-HT and 5-hydroxy indole acetic acid (5-HIAA) in the ileum was increased in AMI group.

\section{Conclusion}

Intestinal dysfunction after AMI may be achieved by decreased intestinal blood perfusion, IDO-related inflammation and the dysfunction of 5-HT metabolic pathway. 


\section{Background}

Acute myocardial infarction (AMI) is one of the most common disease with high morbidity, mortality and disability, which has attracted wide attentions of clinical practice. In addition to chest pain and other typical manifestations, AMI often accompanies intestinal dysfunction, manifested as abdominal pain or distention, diarrhea or constipation, all of which trouble the patients' life quality [1, 2]. However, the mechanism of intestinal dysfunction after AMI has not been fully illuminated, which limits the treatment.

Reports have found intestinal dysfunction was partly attributed to impaired intestinal barrier[3]. In our previous study, after AMI, it was found that the intestinal wall became thin, dropsical, congestive and even hemorrhagic points scattered on the membrane in model rats, which demonstrated visualizedly the occurrence of barrier function failure and inflammatory response in intestine[4]. Being back to upstream, mechanical or chemical stimulations including ischemia may be the sensitive trigger of intestinal barrier dysfunction[1,5]. However, the further mechanism has not been clarified.

Ischemia has been known as one of the basic pathological response in AMI, following which diverse inflammatory cells are activated[1]. Besides T, B or NK cell[6.7], molecular biology researches have found that Indoleamine 2, 3-dioxygenase (IDO) as an indispensable target in intestinal inflammatory diseases participated in the intestinal immune, motility, sensation, ect[8]. Previous reports have been demonstrated besides mediating the inflammatory response, IDO can affect nutrition metabolism, especially the tryptophan (TRP) metabolism[9.10]. Changes in IDO activity can affect TRP metabolism through kynurenine (Kyn) pathway and 5-HT pathway, in which 5-HT has been known as one of the most important neurotransmitters and inflammatory mediators participating in the regulation of intestinal function[11, 12].

The above all should cause us to think, in intestinal dysfuncion after AMI, whether the intestinal ischemia, changes IDO activity and 5-HT pathway was incontrovertible. Further how is the relationship of IDO and 5-HT pathway, the both jointly mediates the inflammatory response after AMI? or IDO related TRP metabolism indirectly influences the 5-HT pathway? All need to be discussed. Anyway, based the previous study, a hypothesis can be proposed, the intesitinal dysfunction after AMI may be related with the intestinal ischemia, and then which may be mediated by the changes of IDO activity and 5-HT metabolism. Here, in the study, we established a rat model of AMI to investigate the changes of intestinal blood supply, IDO and 5-HT metabolism and discuss the possible mechanism of intestinal dysfunction after AMI.

\section{Methods}

\section{Experiment protocol}

The experimental flow chart was as follows, the entire material collection was completed within 3 days. (Fig. 1) 


\section{Animals}

A total of 24 male Wistar rats (body weight 250-280 g) were involved in the trial. All the experiments were approved and supervised by the Animal Care and Use Committee of the Beijing University of Traditional Chinese Medicine. The rats were maintained in a temperature controlled room $\left(18-24^{\circ} \mathrm{C}\right)$ and relative humidity (45-65\%) with a 12-hour light/dark cycle (lights on 7:00-19:00). Animals were allowed free access to food and water. All the experimental animals were approved and supervised by the local ethical review committee on animal care of the Beijing University of Chinese Medicine (2012BZHYLL01034).

\section{Establishment Of The AMI Model}

Rats were divided into three groups randomly, including AMI group $(n=10)$, Sham group $(n=8)$, N group $(n=6)$. Wistar rats were established a acute myocardial infarction model with ligating the left anterior descending artery (LAD) without ventilator assisted[13]. Briefly, Wistar rats were anesthetized with $3 \%$ pentobarbital sodium by intraperitoneal injection $(45 \mathrm{mg} / \mathrm{kg})$. and positioned face up on the operating table. A baseline surface electrocardiogram was recorded using a biological signaling quantitative analysis system (sp-2006, Beijing Softron Biotechnology Ltd., Beijing, China). Rats in AMI group were thorax opened on the left 3-4 intercostal space to expose the heart, the left anterior descending coronary artery (LAD) was ligated at 2-3 mm from the starting point of the left coronary artery between the cone of the pulmonary artery and the left atrial appendage, and then the heart was returned to the thoracic cavity, then the chest and skin were closed and sterilized with iodophor. Rats in sham group underwent all of the same procedures detailed above except for the ligation step. Rats in normal group were given any operation treatment. To prevent infections, rats in AMI and sham groups were injected with $80,000 \mathrm{U} / \mathrm{d}$ penicillin sodium during the 3 days after operation. Surface electrocardiogram (ECG) in AMI and sham groups were recorded after operation, 30 minutes after operation, 4 weeks after operation, postoperation the elevated ST-segment indicated that the model was established successfully.

\section{Echocardiography}

Echocardiography was performed as previously in all rats at 4 weeks after surgery[14-16]. The rats were anesthetized with $3 \%$ pentobarbital sodium by intraperitoneal injection $(45 \mathrm{mg} / \mathrm{kg})$, fixed to the operating table and the hair in the chest area was shaved. Left ventricular internal diameters of systole(LVIDs), Left ventricular internal diameters of diastole (LVIDd) and Interventricular septal thickness at end diastole (IVSd) were measured by the small animals ultrasonic imaging system (Weisheng co, LTD, China), application of $10 \mathrm{~s}$ frequency probe, $10 \mathrm{MHZ}$ frequency, angle of probe 15 to $30^{\circ}, 2-3 \mathrm{~cm}$ deep. And Left ventricular ejection fraction (LVEF) and Fractional shortening (FS) were calculated.

\section{Detection Of Intestinal Propulsive Rate}


As the previous methods[17-19], rats were fasted for 24 hours before the experiment and free water. Each rat was given a nutritional semi-solid paste of $1 \mathrm{ml} / 100 \mathrm{~g}$ (a nutritional semi-solid paste: $10 \mathrm{~g}$ carboxymethyl cellulose sodium, $16 \mathrm{~g}$ milk powder, $8 \mathrm{~g}$ sugar, $8 \mathrm{~g}$ starch and $2 \mathrm{~g}$ activated carbon were dissolved into $250 \mathrm{ml}$ distilled water and stirred evenly for use). After $20 \mathrm{~min}$, the rats were sacrificed, and the small intestine was separated from the pylorus to the ileocecal section, and the intestine was laid flat on the operating table without traction. The activated carbon propulsion distance and total intestinal length were measured. Intestinal propulsive rate $=$ activated carbon propulsion distance/total intestinal length $* 100 \%$.

\section{Tissue Harvesting And Processing}

The left ventricle was cut perpendicularly to the midpoint of the long axis of the rat heart, and the myocardial tissue at the apex of the heart was retained for $6 \times 6 \times 6 \mathrm{~mm}$, and the intestinal segment at the end of the ileum was retained for $2 \mathrm{~cm}$.

The obtained myocardial tissues and ileac tissue $(1 \mathrm{~cm})$ were fixed in $4 \%$ paraformaldehyde solution. After the tissue is dehydrated, transparent and embedded in paraffin, the wax block is sectioned continuously (5 um) for use. The remaining ileac tissues $(1 \mathrm{~cm})$ were preserved at $-80^{\circ} \mathrm{C}$.

\section{Hematoxylin-eosin (HE) Staining For Myocardial And lleac Tissue}

The main steps were as follows: (1) Dewaxing: dewaxing by 100\% xylene for 15 min, 3 times, soaking in anhydrous ethanol, $95 \%, 80 \%, 75 \%$ alcohol successively for $3 \mathrm{~min}$, washing with pure water for reserve; (2) Hematoxylin staining: Immersing in Harris hematoxylin solution for 2 min, washing with pure water

for $3 \mathrm{~min}$; (3) Color separation: differentiating by $0.5 \%$ alcohol with hydrochloric acid for $5 \mathrm{sec}$, and putting in carbonate saturated solution for $10 \mathrm{sec}$, then washing with pure water for $2 \mathrm{~min}$; (4) Eosin staining: Immersing in $0.5 \%$ eosin solution for $3 \mathrm{~min}$; (5) Decolorization and transparency: decolorization with $80 \%$, $90 \%$, anhydrous ethanol, and transparency with xylene for 15 min, 3 times; (6) Sealing: sealing the cover glass with neutral balsam; Under light microscope (OLYMPUS co., LTD, Japan), myocardial tissue and distal ileac tissue were selected for observation.

\section{Laser speckle technique for measuring mesenteric blood flow[20]}

Rats were anesthetized with $3 \%$ pentobarbital sodium by intraperitoneal injection $(45 \mathrm{mg} / \mathrm{kg})$. the injection dose is $25 \mathrm{mg} / \mathrm{kg}$ ), shaved and incised in the middle of the abdomen ( $1.5 \mathrm{~cm}$ incision), and $10 \mathrm{~cm}$ of the intestinal segment with mesentery at the end of the ileum were gently took out from the abdominal cavity and placed on a thermostatic plate $\left(37.7^{\circ} \mathrm{C}\right)$. Ringer's solution (a mixture of phosphate buffered saline and $1 \%$ calf serum albumin, $37^{\circ} \mathrm{C}, \mathrm{pH} 7.4$ ) was continuously showered on the mesentery. The laser 
speckle blood flow video detection system (PERIMED co., LTD, Sweden) emitted the laser light from the helium-neon beam and the laser light scattered on the test site to detect blood flow.

\section{Immunohistochemistry For IDO Expression}

The ileum tissue were dehydrated and embedded in paraffin following routine methods. The paraffin sections were removed paraffin, and then immersed in the distilled water following routine methods. Afterwards, rinsing the paraffin sections in PBS, and then blocked with goat serum. Incubated at room temperature, colouration with $\mathrm{DAB}$, counterstain with hematoxylin, mounted with neutral gum. The standard of IDO-positive cells were stained with brown or yellow-brown, the optical density value (OD) of IDO were analyzed use Image-Pro Plus software. Statistics under high magnification (20x), and the average was taken.

\section{High-performance Liquid Chromatography For TRP Metabolism}

Rat plasma sample preparation: Took $4 \mathrm{ml}$ of blood from the abdominal aorta of the rat, put it into a centrifuge tube with heparin sodium, centrifuged at $(900 \mathrm{r} / \mathrm{min}), 10 \mathrm{~min}$, took the supernatant; Then, took $100 \mathrm{ul}$ of the supernatant, added the same volume (100 ul) 5M perchloric acid solution to vortex mixed for $30 \mathrm{~s}$, centrifuged $(14000 \mathrm{r} / \mathrm{min})$ at $4{ }^{\circ} \mathrm{C}$ for $10 \mathrm{~min}$, took the supernatant and waited for measurement.

Rat ileum samples preparation: Took the length of $1 \mathrm{~cm}$ ileum tissue, it was cut along the edge of the mesentery on the ice tray, removed the impurities and blood with ice-cold physiological saline, dried it with filter paper, accurately weighted and recorded. Added $1 \mathrm{~g} / 5 \mathrm{ml}$ of pre-ice-cooled $0.5 \mathrm{M}$ perchloric acid solution, homogenized, centrifuged $(5000 \mathrm{r} / \mathrm{min})$ at $4^{\circ} \mathrm{C}$ for $10 \mathrm{~min}$, took $100 \mu \mathrm{L}$ of supernatant, added $100 \mu \mathrm{L}$ of $0.5 \mathrm{M}$ perchloric acid solution, and vortex to mixed for $30 \mathrm{~s}$. Centrifuged $(14000 \mathrm{r} / \mathrm{min})$ at $4^{\circ} \mathrm{C}$ for 10 minutes, and retained the supernatant for measurement.

The column conditions were ZORBAX Eclipse AAA column $(4.6 \times 150 \mathrm{~mm}, 5 \mu \mathrm{m})$, at $225 \mathrm{~nm}$, used ultraviolet detected Kyn; at 280 nm, 340 nm, 254 nm, 404 nm, used fluorescence detected 5-HT, Tryptophan(TRP), 5-hydroxyindole acetic acid(5-HIAA), kynurenic acid(KynA) respectively. The date were analyzed on an Agilent ChemStation (Agilent co., LTD, USA).

\section{Statistical analysis}

Statistical analysis was performed with SPSS 17.0 software(SPSS, Chicago, IL, USA). Date were represented as mean \pm S.E.M, all data were tested for normality and homogeneity of variance firstly, oneway analysis of variance(ANOVA) was used for comparison among the three groups. If there were 
statistical differences among the three groups, then the LSD-t test was used to compare the mean between the two groups, a value $P<0.05$ was considered statistically.

\section{Results}

\section{General condition of rats after operation}

In AMI and Sham group, 6 survived from the surgery in AMI group and Sham group respectively, the died rats were mainly caused by intraoperative or post-operative arrhythmia. Rats in AMI group had shown reducing diet, not shiny hair, reduction of activity, slower weight growth, and cyanosis of distal limbs. No signs of lung infection or pneumothorax or pathological state of postoperative inflammation had been investigated.

\section{Changes In Body Surface ECG}

The successful criterion for AMI is that the ECG shows ST segment elevation in lead $\nabla$ for more than 30 minutes. The ST segment elevation also appeared in the sham group, but the elevation range was less than that in the AMI group, and it was close to the preoperative level after 30 minutes, pathological Q wave appeared at 4 weeks after surgery in the AMI group. (Fig. 2)

\section{Changes Of Myocardial Tissue After AMI}

At 4 weeks after AMI, the ventricular wall of the infarcted area of the rats became thin and white; the sham group had normal heart shape and dark red. HE staining showed that the normal myocardial cells were arranged in an orderly manner; the infarcted myocardium was replaced by a large number of fibrous tissues, the structure was disordered, the myocardial fibers were swollen, the horizontal stripes disappeare and a large number of inflammatory cells infiltrated. (Fig. 3)

\section{Changes Of lleum Tissue After AMI}

At 4 weeks after AMI, the intestinal wall of rats became thin and edema, the mucosa became scattered around the bleeding point. the sham group had normal shape. HE staining showed that intestinal mucosa was hyperemia, edema, and it was infiltrated by a large number of neutrophils. (Fig. 4)

\section{Changes Of Echocardiogram After AMl}

At 4 weeks after surgery, LVIDs, LVIDd, and IVSd were parameters that reflected the heart structure of rats. LVIDs, LVIDd, and IVSd among the three groups were statistically significant at 4 weeks after surgery $(P<0.05)$. Further compared with the Sham group and the N group, LVIDs and LVIDd in the AMI group 
increased significantly $(P<0.01)$, and IVSd decreased significantly $(P<0.01)$. Compared with the $\mathrm{N}$ group, LVIDs, LVIDd and IVSd were not statistically significant in the Sham group (P>0.05). (Fig. 5)

LVEF and FS were parameters that reflected systolic function. LVEF and FS among the three groups were statistically significant at 4 weeks after surgery $(P<0.05)$. Further compared with the Sham group and the $\mathrm{N}$ group, LVEF and FS values in the AMI group were significantly decreased $(P<0.01)$. Compared with the $\mathrm{N}$ group, the Sham group was not statistically significant ( $P>0.05)$. (Fig. 5)

\section{Measurement Of Intestinal Propulsive Rate In Rats After AMI}

Intestinal propulsive rate was statistically significant among the three groups $(P<0.05)$. Further comparison, the AMI group had higher intestinal propulsive rate than the Sham group and the $\mathrm{N}$ group $(P<0.05)$. Compared with the $\mathrm{N}$ group, the Sham group was not statistically significant $(P>0.05)$. (Fig. 6)

\section{Measurement of mesenteric blood flow in rat ileum after AMI}

The mesenteric blood flow in the distal ileum of rats was statistically significant at 4 weeks after operation $(P<0.05)$. Further pairwise comparison, the mesenteric blood flow in the AMI group was significantly lower than that in the Sham group and the $\mathrm{N}$ group $(P<0.05)$. Compared with the $\mathrm{N}$ group, the Sham group was not statistically significant (P>0.05). (Fig. 7)

\section{Expression Of IDO}

IDO was mainly expressed in the mucous layer of the distal ileum. The optical density of IDO among the three groups was statistically significant $(P<0.05)$. Further pairwise comparison, compared with the Sham group and the $\mathrm{N}$ group, the optical density of IDO increased in AMI group $(P<0.01)$. The Sham group had no statistical significance compared with the $\mathrm{N}$ group(P>0.05). (Fig. 8)

\section{TRP and Its metabolites in the plasma}

The contents of TRP, 5-HIAA, Kyn, and KynA in the plasma among the three groups were not statistically significant $(P>0.05)$. The $5-\mathrm{HT}$ content among the three groups was statistically significant $(P<0.05)$. Further pairwise comparison, compared with the Sham group and the $\mathrm{N}$ group, the 5 -HT content increased $(P<0.05)$. The Sham group had no statistical significance compared with the $\mathrm{N}$ group $(P>0.05)$. (Fig. 9)

\section{TRP And Its Metabolites In The lleum}


TRP content in the distal ileum among the three groups was not statistically significant $(P>0.05)$. The content of 5-HT and 5-HIAA in the distal ileum was statistically significant $(P<0.05)$. Further pairwise comparison, the content of 5-HT and 5-HIAA increased in AMI group compared with the Sham group and the $\mathrm{N}$ group $(P<0.05)$. Compared with the $\mathrm{N}$ group and the Sham group, there was not statistically significant ( $P>0.05)$. (Fig. 10)

\section{Discussion}

In the study, we established a model of AMI. At 4 weeks after AMI, the major lesions in the myocardial cell, ventricular remodeling and decreased cardiac ejection were observed. Meanwhile, intestinal mucosa was destructed and was changed by inflammation, intestinal motility increased. Further, it was demonstrated that mesenteric blood flow of the distal ileum decreased, the expression of IDO increased, and the level of 5-HT in plasma and ileum increased, which suggested mesenteric ischemia, abnormalities in IDO activity and 5-HT pathway may participate the intestinal dysfunction after AMI.

\section{Intestinal Dysfunction After AMI}

AMI has been known as a severe cardiovascular disease with a high incidence worldwide, whose pathological basis was myocardial necrosis caused by persistent myocardial ischemia and hypoxia. There were estimated that $31 \%$ of people die from cardiovascular disease worldwide, of which myocardial infarction is the main cause of death[21]. After myocardial infarction, multiple systems can produce concurrent symptoms, especially the digestive system, which have troubled the patients[1]. And the dysfunctions of intestinal microenvironment including the microbial metabolism, intestinal epithelium barrier are the significative pathological characteristics in myocardial infarction[1, 2, 22, 23]. Further, Zhou $\mathrm{X}$ et al have reported that in ST-segment elevation myocardial infarction patients, intestinal mucosal injury and intestinal microbiota translocation has been investigated and the higher LPS and d-lactate predicted a poorer cardiovascular outcome, further, after application of gut microbial translocation inhibitor chould reduce infarct size[1]. At the same time, the study found that the performance of electrocardiogram in patients with myocardial infarction can be improved by the improvement of gastrointestinal function[1, 24]. All of which demostrated that the intestinal dysfunction palyed a key role in the prognosis of myocardial infarction. However, the mechanisms of gastrointestinal dysfunction has not been fully elucidated and need to be clarified.

In our study, we successfully mimicked the AMI state which can be demonstrated by the ST segment

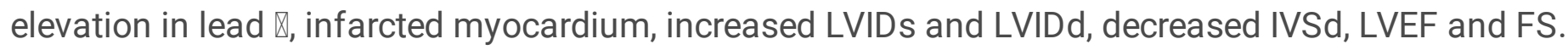
Besides, after AMI at 4 weeks, we observed obviously intestinal flatulence, intestinal wall edema, and bleeding points scattered in the mucosa. In our previous study, the intestinal pathology has shown that the intestinal mucosa was destroyed and an inflammatory reaction occurred. The villi of the small intestine were transected, shortened, fused significantly, and a large number of neutrophils invaded the mucosa and submucosa[4], which was validated again in the study. Besides, Intestinal motility test had 
shown that intestinal propulsive rate was significantly increased. All the above suggested that the intestinal structure and function of rats changed significantly after AMI. This has a certain degree of consistency with research reports, indicating that gastrointestinal disorders occur after AMI[1] .

\section{Mesenteric Ischemia After AMI}

The normal intestinal mucosa has abundant blood supply, enough area to maintain the normal mucosal barrier function and resist the invasion of bacteria and toxins in the intestinal tract. The supply of adequate nutrients and oxygen was the basis to ensure the normal structure and function of the intestinal mucosa. Intestinal ischemia and hypoxia interrupted the source of nutrients in intestinal tissue cells, resulting the lack of energy in cell metabolism and substance synthesis, and leading to the generation of a large number of free radicals, the damage to intestinal tissue, and the increase of epithelial permeability[1, 24].

In the study, it has been showed that through dynamic observation, we found that hypoperfusion in the mesentery continued to 4 weeks after the operation, suggesting that decreased mesenteric hypoperfusion may play an important role in intestinal dysfunction after AMI.

\section{Inflammatory pathological changes and IDO activation after AMI}

Mesenteric hypoperfusion could cause the damage of the intestinal vascular endothelial, the increase of intestinal epithelial permeability, and the gather of inflammatory mediators, as IFN-a,TFN-a,IL-1[25-27]. Besides, as one of inflammatory cells, IDO could also significantly increased in a state of inflammation or infection[28].

In our experiment, 4 weeks after the operation, intestinal wall was edematous and the lumen was flatulent. Intestinal HE staining has shown intestinal mucosa was destroyed and a large number of neutrophils gathered in the mucosa and submucosa. Meanwhile, Immunohistochemistry showed increased expression of IDO in ileum mucosa, which was partly consistent with reports of increased expression of IDO in patients with crohn's disease and ulcerative colitis[29], and suggested that there was an inflammatory response during intestinal hypoperfusion after AMI, in which IDO participated.

\section{Abnormalities In 5-HT Pathway After AMI}

Besides including in the inflammatory response, IDO has been known as a key rate limiting enzyme in the metabolic pathway of TRP. TRP is an important precursor of 5-HT and Kyn, and IDO mainly mediates TRP metabolism through Kyn pathway, which can affect TRP metabolism through the 5-HT pathway[27]. 5-HT is an important peptide in the intestine, which is closely related to intestinal motility, secretion and sensation. In our previous study, it also had been demonstrated that abnormalities of 5-HT metabolism in brain-gut axis played a key role in the depression and gastrointestinal dysfunction after AMI[30]. $90 \%$ of $5-\mathrm{HT}$ in the the body is synthesized by chromaffin cells in the intestine. TRP produces 5 - 
hydroxytryptophan by tryptophan hydroxylase-1 (THP-1) and then 5-hydroxytryptamine acid decarboxylase catalyzes 5-hydroxytryptophan to 5-HT. 5-HT exerts biological effects by binding to specific receptors $^{(11)}$ and terminates the activity by $5-\mathrm{HT}$ selective reuptake transporter (SERT). Generally, 5 -HT is degraded to 5-hydroxyindoleacetic acid (5-HIAA) by monoamine oxidase (MAO). In our experiments, we obtained that $5-\mathrm{HT}$ content in the plasma and ileum in the model group was significantly increased, which might be an important scientific explanation of the increased intestinal motility.

However, in our study, we noted a thought-provoking result, which was in contradiction with the theory "serotonin hypothesis". Serotonin hypothesis indicates that TRP diverts from 5-HT to synthesize Kyn, which is a well-known theory in neurogenic disease as depression[31, 32]. However, in the study, when IDO increased, TRP level had no significant in plasma among the three groups, while the 5-HT in plasma increased, and Kyn and KynA in plasma has no statistical significance, suggesting that the relationship of IDO changes and 5-HT metabolism is different with the "serotonin hypothesis"after AMI. The possible reasons for the increase of IDO and 5-HT in the same direction were analyzed as follows:1. IDO was an inflammatory mediator and 5-HT was an anti-inflammatory mediators: Previous study ${ }^{[11]}$ has been shown that in the intestinal tissue, 5-HT had anti-inflammatory effect, which not only reduced enteric nervous inflammation and damage, but also promoted the nerve regeneration. This reminded the increase of 5-HT after AMI was probably the feedback response of the body to cope with the stress and inflammatory reaction of myocardial infarction, which was in order to reduce the intestinal mucosa and nerve damage[11, 33]; 2. TRP accumulation: TRP is an important amino acid ingested by the intestine from food. It has been reported that oxidative stress could result the accumulation of TRP in intestine[33-36]. In our study, it was found that TRP did not decrease due to the increase of 5-HT, suggesting that stress and inflammatory response after AMI might cause the accumulation of TRP, which could ensure the metabolic requirements. In summary, the increase of IDO had not inhibited the TRP/5-HT metabolic pathway, which may be associated with the inflammatory response, so the results were reasonable, but the further mechanisms needed to be discussed.

\section{Conclusion}

Taken together, Intestinal dysfunction in rats after AMI may be achieved by decreased intestinal blood perfusion, IDO-related inflammation and 5-HT metabolic pathway. Further 5-HT metabolism may be associated with the inflammatory response, in which IDO may participate, at least in part.

\section{Abbreviations}

AMI: acute myocardial infarction; $\mathrm{N}$ group: normal group; LAD: left anterior descending artery; ECG: body surface electrocardiogram; IDO: Indoleamine 2, 3-dioxygenase; 5-HT: 5-hydroxytryptamine; LVIDd: Left ventricular internal diameters of diastole; IVSd: Interventricular septal thickness at end diastole; LVEF: Left ventricular ejection fraction; FS: Fractional shortening; TRP: tryptophan; Kyn: kynurenine; LAD: left anterior descending artery. 


\section{Declarations}

\section{Authors' contributions}

YGW and YFW conceived and designed the study. XFL conducted the experiments. XFL and PPZ performed the statistical analysis, and designed the figures. XFL and PPZ wrote the manuscript. YGW and YFW reviewed and edited the manuscript. All authors read and approved the final manuscript.

\section{Author details}

${ }^{1}$ Digestive Disease Center, Beijing Hospital of Traditional Chinese Medicine Affiliated to Capital Medical University, Beijing, China. ${ }^{2}$ Hebei College of Traditional Chinese Medicine,Shijiazhuang, Hebei, China.

${ }^{3}$ Department of Gastroenterology, Affiliated Hospital of Hebei College of Traditional Chinese Medicine, Shijiazhuang, Hebei, China. ${ }^{4}$ Department of Nephropathy, Beijing Hospital of Traditional Chinese MedicineAffiliated to Capital Medical University, Beijing, China.

\section{Acknowledgements}

Not applicable

\section{Competing interests}

The authors declare that they have no competing interests.

\section{Availability of data and materials}

I agree to share my data and materials

\section{Consent for publication}

Not applicable.

\section{Ethics approval and consent to participate}

Animals were allowed free access to food and water. All the experimental animals were approved and supervised by the local ethical review committee on animal care of the Beijing University of Chinese Medicine (2012BZHYLL01034).

\section{Funding}

Not applicable

\section{References}


1. Zhou X, Li J, Guo J, Geng B, Ji W, Zhao Q, et al. Gut-dependent microbial translocation induces inflammation and cardiovascular events after ST-elevation myocardial infarction. Microbiome. 2018;6(1):66.

2. Mao Y, Wang SQ, Mao XB, Zeng QT, Li YS. Intestinal barrier function in patients with acute myocardial infarction and the therapeutic effect of glutamine. Int J Cardiol. 2011;146(3):432-3.

3. Liu L, Yao J, Li Z, Zu G, Feng D, Li Y, et al. miR-381-3p knockdown improves intestinal epithelial proliferation and barrier function after intestinal ischemia/reperfusion injury by targeting nurr1. Cell Death Dis. 2018;9(3):411.

4. Lu X, Wang Y, Wang Y. Gastrointestinal Disorder in Myocardial Infarction Rats Based on Hemodynamic Change. Journal of Medical Research. 2014;43(07):144-7.

5. He W, Wang Y, Wang P, Wang F. Intestinal barrier dysfunction in severe burn injury. Burns Trauma. 2019;7:24.

6. Frangogiannis NG. Regulation of the inflammatory response in cardiac repair. Circ Res. 2012;110(1):159-73.

7. Frangogiannis NG. The inflammatory response in myocardial injury, repair, and remodelling. Nat Rev Cardiol. 2014;11(5):255-65.

8. Ciorba MA. Indoleamine 2,3 dioxygenase in intestinal disease. Curr Opin Gastroenterol. 2013;29(2):146-52.

9. Messaoud A, Mensi R, Douki W, Neffati F, Najjar MF, Gobbi G, et al. Reduced peripheral availability of tryptophan and increased activation of the kynurenine pathway and cortisol correlate with major depression and suicide. World J Biol Psychiatry. 2019;20(9):703-11.

10. Liu W, Sheng H, Xu Y, Liu Y, Lu J, Ni X. Swimming exercise ameliorates depression-like behavior in chronically stressed rats: relevant to proinflammatory cytokines and IDO activation. Behav Brain Res. 2013;242:110-6.

11. Gershon MD. 5-Hydroxytryptamine (serotonin) in the gastrointestinal tract. Curr Opin Endocrinol Diabetes Obes. 2013;20(1):14-21.

12. Ghia JE, Li N, Wang H, Collins M, Deng Y, El-Sharkawy RT, et al. Serotonin has a key role in pathogenesis of experimental colitis. Gastroenterology. 2009;137(5):1649-60.

13. Trueblood NA, Xie Z, Communal C, Sam F, Ngoy S, Liaw L, et al. Exaggerated left ventricular dilation and reduced collagen deposition after myocardial infarction in mice lacking osteopontin. Circ Res. 2001;88(10):1080-7.

14. Chen J, Zhan Y, Wang Y, Han D, Tao B, Luo Z, et al. Chitosan/silk fibroin modified nanofibrous patches with mesenchymal stem cells prevent heart remodeling post-myocardial infarction in rats. Acta Biomater. 2018;80:154-68.

15. Nozawa E, Kanashiro RM, Murad N, Carvalho AC, Cravo SL, Campos O, et al. Performance of twodimensional Doppler echocardiography for the assessment of infarct size and left ventricular function in rats. Braz J Med Biol Res. 2006;39(5):687-95. 
16. Burrell LM, Chan R, Phillips PA, Calafiore P, Tonkin AM, Johnston Cl. Validation of an echocardiographic assessment of cardiac function following moderate size myocardial infarction in the rat. Clin Exp Pharmacol Physiol. 1996;23(6-7):570-2.

17. Matsuda H, Shimoda H, Ninomiya K, Yoshikawa M. Inhibitory mechanism of costunolide, a sesquiterpene lactone isolated from Laurus nobilis, on blood-ethanol elevation in rats: involvement of inhibition of gastric emptying and increase in gastric juice secretion. Alcohol Alcohol. 2002;37(2):121-7.

18. Howd RA, Adamovics A, Palekar A: Naloxone and intestinal motility. Experientia: 1978;34:13101311.

19. Nakamura R, Kato J, Kitamura K, Onitsuka H, Imamura T, Marutsuka K, et al. Beneficial effects of adrenomedullin on left ventricular remodeling after myocardial infarction in rats. Cardiovasc Res. 2002;56(3):373-80.

20. Zheng C, Lau LW, Cha J. Dual-display laparoscopic laser speckle contrast imaging for real-time surgical assistance. Biomed Opt Express. 2018;9(12):5962-81.

21. Roger VL, Go AS, Lloyd-Jones DM, Benjamin EJ, Berry JD, Borden WB, et al. Heart disease and stroke statistics-2012 update: a report from the American Heart Association. Circulation. 2012;125(1):e2220.

22. Deng D, Liu L, Xu G, Gan J, Shen Y, Shi Y, et al. Epidemiology and Serum Metabolic Characteristics of Acute Myocardial Infarction Patients in Chest Pain Centers. Iran J Public Health. 2018;47(7):101729.

23. Wu ZX, Li SF, Chen H, Song JX, Gao YF, Zhang F, et al. The changes of gut microbiota after acute myocardial infarction in rats. PLoS One. 2017;12(7):e0180717.

24. Herath HM, Thushara Matthias A, Keragala BS, Udeshika WA, Kulatunga A. Gastric dilatation and intestinal obstruction mimicking acute coronary syndrome with dynamic electrocardiographic changes. BMC Cardiovasc Disord. 2016;16(1):245.

25. Altshuler AE, Lamadrid I, Li D, Ma SR, Kurre L, Schmid-Schönbein GW, et al. Transmural intestinal wall permeability in severe ischemia after enteral protease inhibition. PLoS One. 2014;9(5):e96655.

26. Ceulemans LJ, Verbeke L, Decuypere JP, Farré R, De Hertogh G, Lenaerts K, et al. Farnesoid X Receptor Activation Attenuates Intestinal Ischemia Reperfusion Injury in Rats. PLoS One. 2017;12(1):e0169331.

27. Binder A, Ali A, Chawla R, Aziz HA, Abbate A, Jovin IS. Myocardial protection from ischemiareperfusion injury post coronary revascularization. Expert Rev Cardiovasc Ther. 2015;13(9):1045-57.

28. Furuzawa-Carballeda J, Fonseca-Camarillo G, Lima G, Yamamoto-Furusho JK. Indoleamine 2,3dioxygenase: expressing cells in inflammatory bowel disease-a cross-sectional study. Clin Dev Immunol. 2013;2013:278035.

29. Ciccocioppo R, Cangemi GC, Kruzliak P, Gallia A, Betti E, Badulli C, et al. Ex vivo immunosuppressive effects of mesenchymal stem cells on Crohn's disease mucosal T cells are largely dependent on indoleamine 2,3-dioxygenase activity and cell-cell contact. Stem Cell Res Ther. 2015;6(1):137. 
30. Lu X, Wang Y, Liu C, Wang Y. Depressive disorder and gastrointestinal dysfunction after myocardial infarct are associated with abnormal tryptophan-5-hydroxytryptamine metabolism in rats. PLoS One. 2017;12(2):e0172339.

31. Jenkins TA, Nguyen JC, Polglaze KE, Bertrand PP. Influence of Tryptophan and Serotonin on Mood and Cognition with a Possible Role of the Gut-Brain Axis. Nutrients. 2016;8(1).

32. Zhou YF, Feng L, Liu XM, Tao X, Wang LS, Zhang MD, et al. Urinary metabolic disturbance in the olfactory bulbectomized rats and the modulatory effects of fluoxetine. Life Sci. 2019;234:116751.

33. Liu MY, Zhang LJ, Zhou YX, Wei WL. 5-Hydroxytryptamine Changes under Different Pretreatments on Rat Models of Myocardial Infarction and/or Depression. Chin Med J (Engl). 2017;130(18):2219-25.

34. Platten M, Nollen EAA, Röhrig UF, Fallarino F, Opitz CA. Tryptophan metabolism as a common therapeutic target in cancer, neurodegeneration and beyond. Nat Rev Drug Discov. 2019;18(5):379401.

35. Gao J, Xu K, Liu H, Liu G, Bai M, Peng C, et al. Impact of the Gut Microbiota on Intestinal Immunity Mediated by Tryptophan Metabolism. Front Cell Infect Microbiol. 2018;8:13.

36. Zelante T, lannitti RG, Cunha C, De Luca A, Giovannini G, Pieraccini G, et al. Tryptophan catabolites from microbiota engage aryl hydrocarbon receptor and balance mucosal reactivity via interleukin-22. Immunity. 2013;39(2):372-85.

\section{Figures}




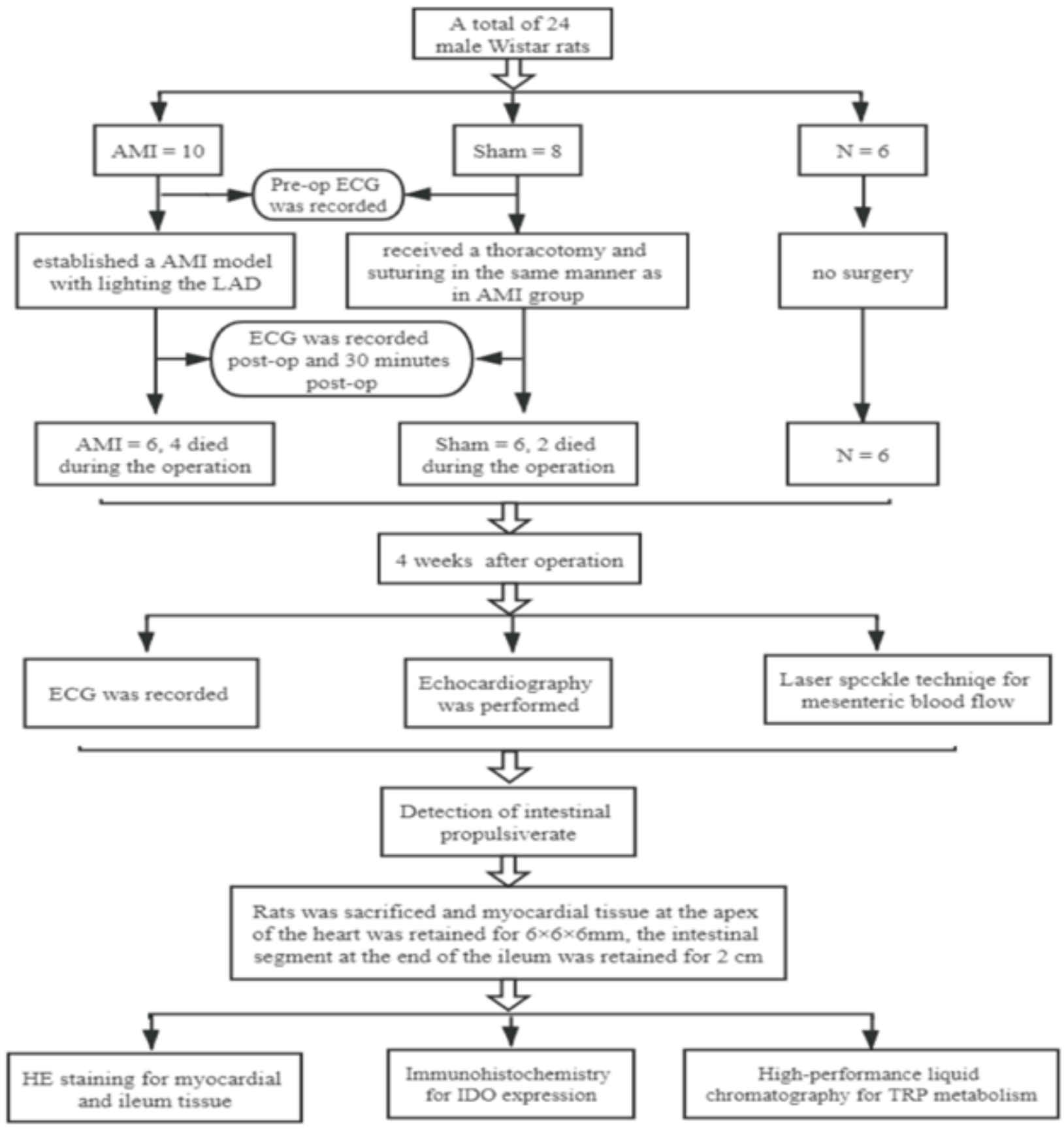

Figure 1

Experimental flowchart 


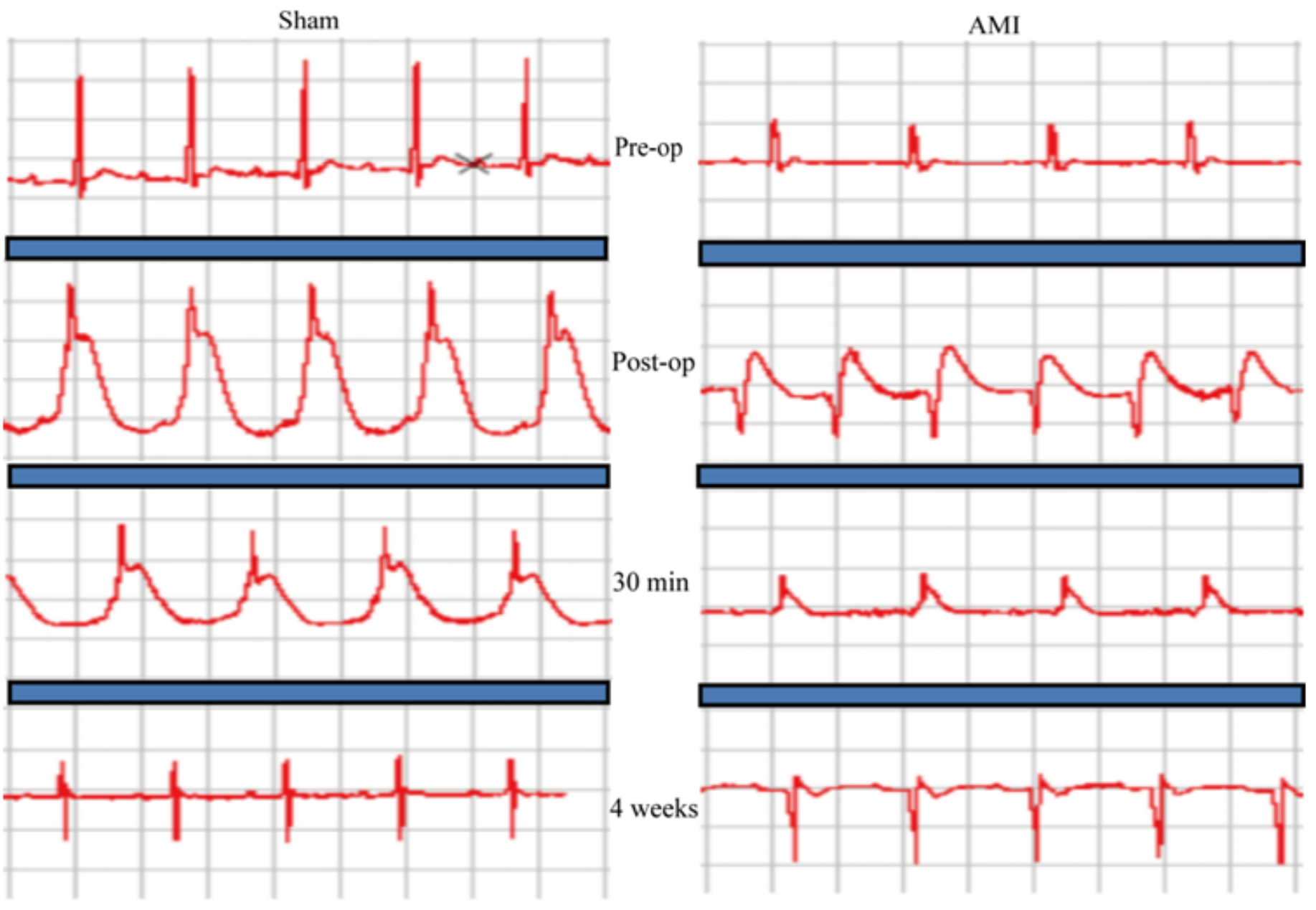

Figure 2

ECG changes at lead II after AMI and sham operation in rats; pathological Q wave appeared at 4 weeks after surgery in the AMI group.
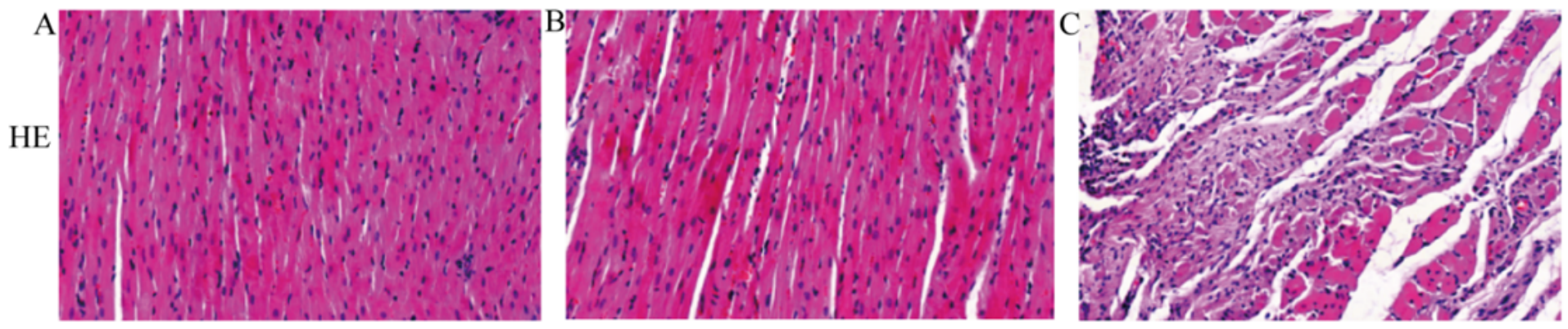

Figure 3

Histopathology changes in heart among the three groups (magnification 20X). (A) N group. (B) Sham group. (C) AMI group. 

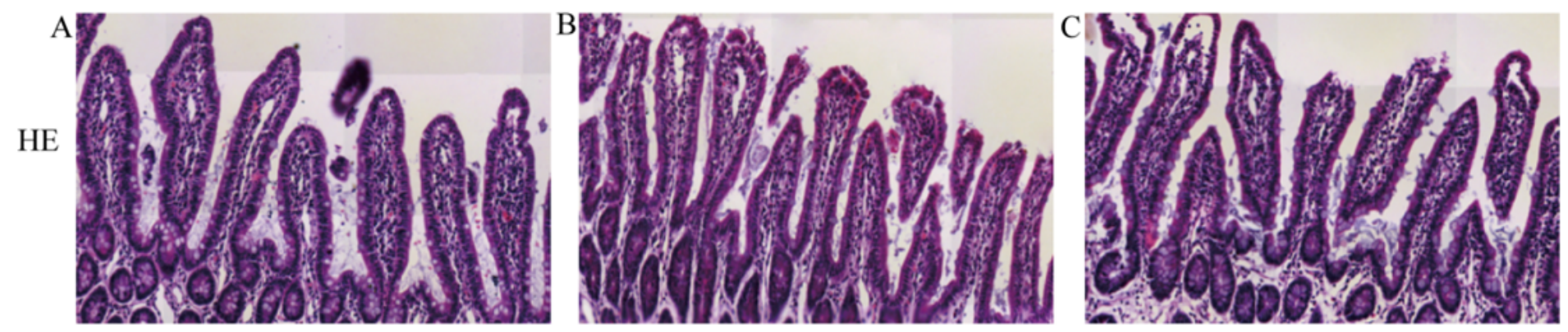

Figure 4

Histopathology changes in ileum tissue among the three groups (magnification 20X). (A) N group. (B) Sham group. (C) AMI group.

A

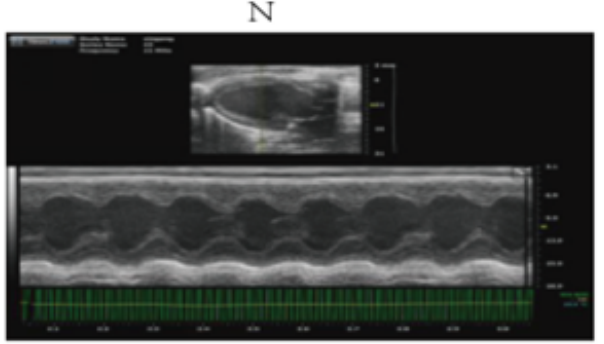

B

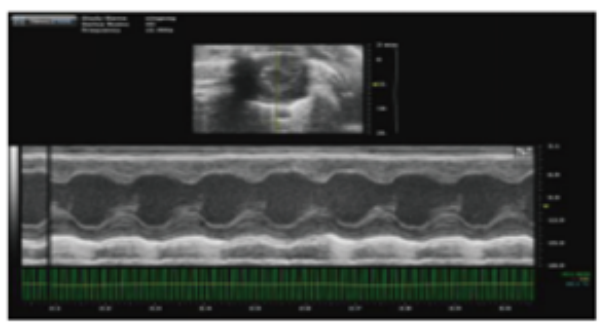

C

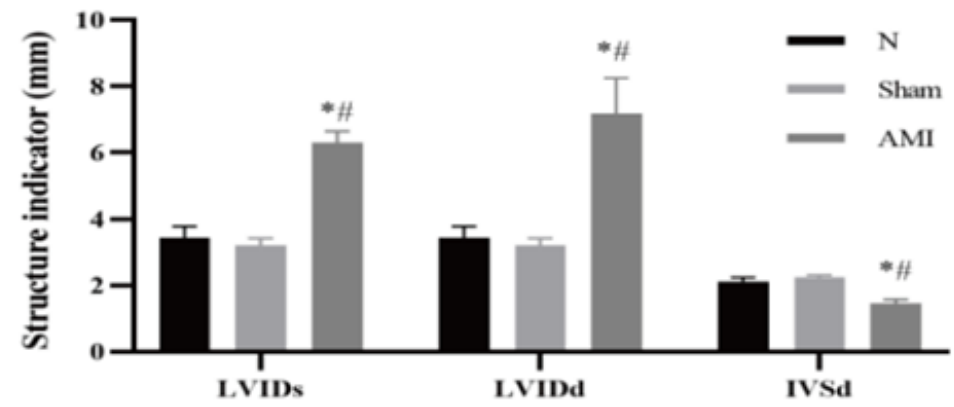

Sham
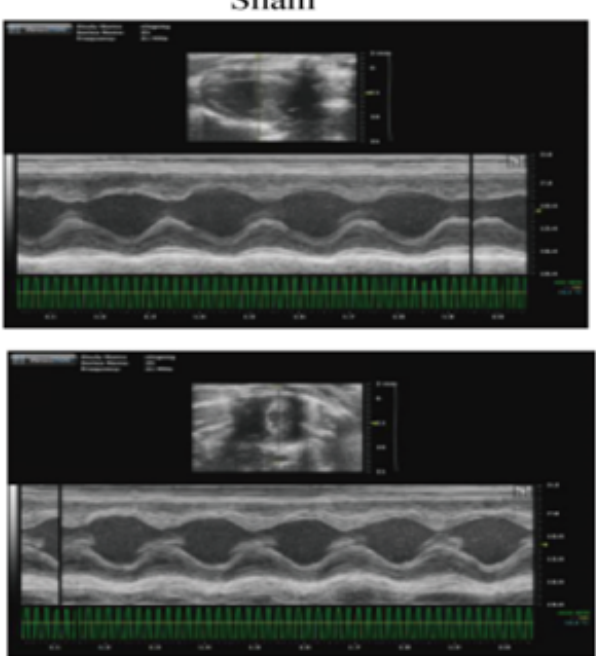

D

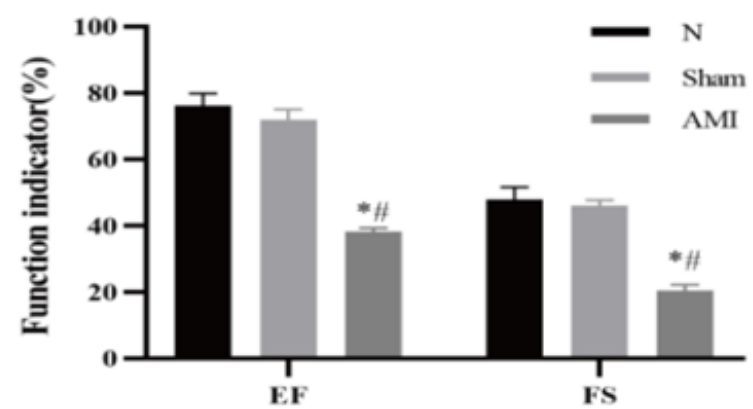

Figure 5

Cardiac function measured by echocardiography (mean $\pm S E M, n=6)$. (A-B)Echocar-diographic pattern of the heart; (C)Structure indicators; (D) Functio-n indicators. * $P<0.01$ vs $N$ group; \# $P<0.01$ vs Sham group. 


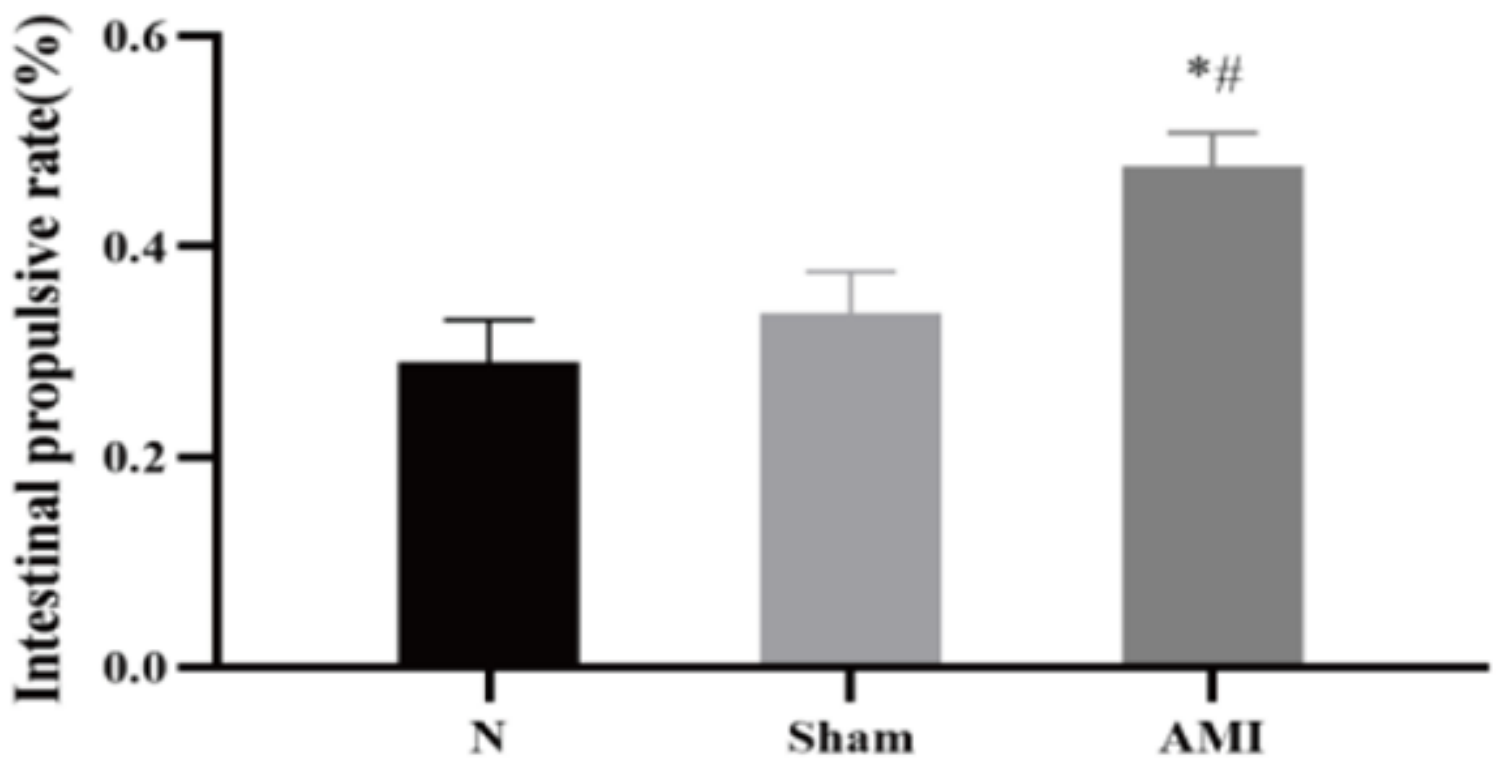

Figure 6

Intestinal propulsive rate (mean $\pm S E M, n=6) .{ }^{*} P<0.01$ vs $N$ group, \# $P<0.05$ vs Sham group. 

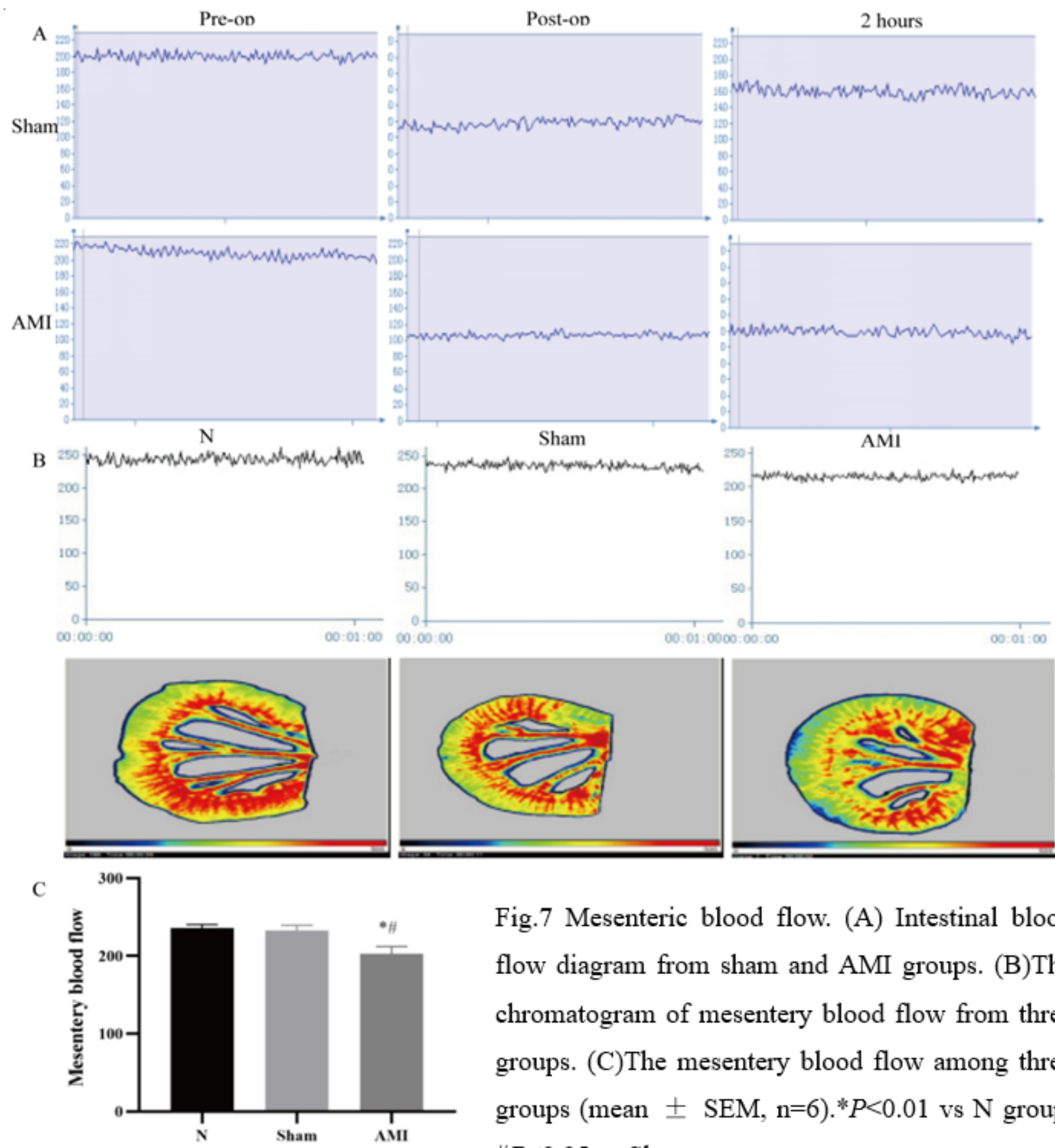

Fig.7 Mesenteric blood flow. (A) Intestinal blood flow diagram from sham and AMI groups. (B)The chromatogram of mesentery blood flow from three groups. (C)The mesentery blood flow among three groups (mean $\pm \mathrm{SEM}, \mathrm{n}=6$ ). ${ }^{*} P<0.01$ vs $\mathrm{N}$ group, $\# P<0.05$ vs Sham group.

\section{Figure 7}

Mesenteric blood flow. (A) Intestinal blood flow diagram from sham and AMI groups. (B)The chromatogram of mesentery blood flow from three groups. (C)The mesentery blood flow among three groups (mean $\pm S E M, n=6$ ). ${ }^{*}<0.01$ vs $N$ group, $\# P<0.05$ vs Sham group. 

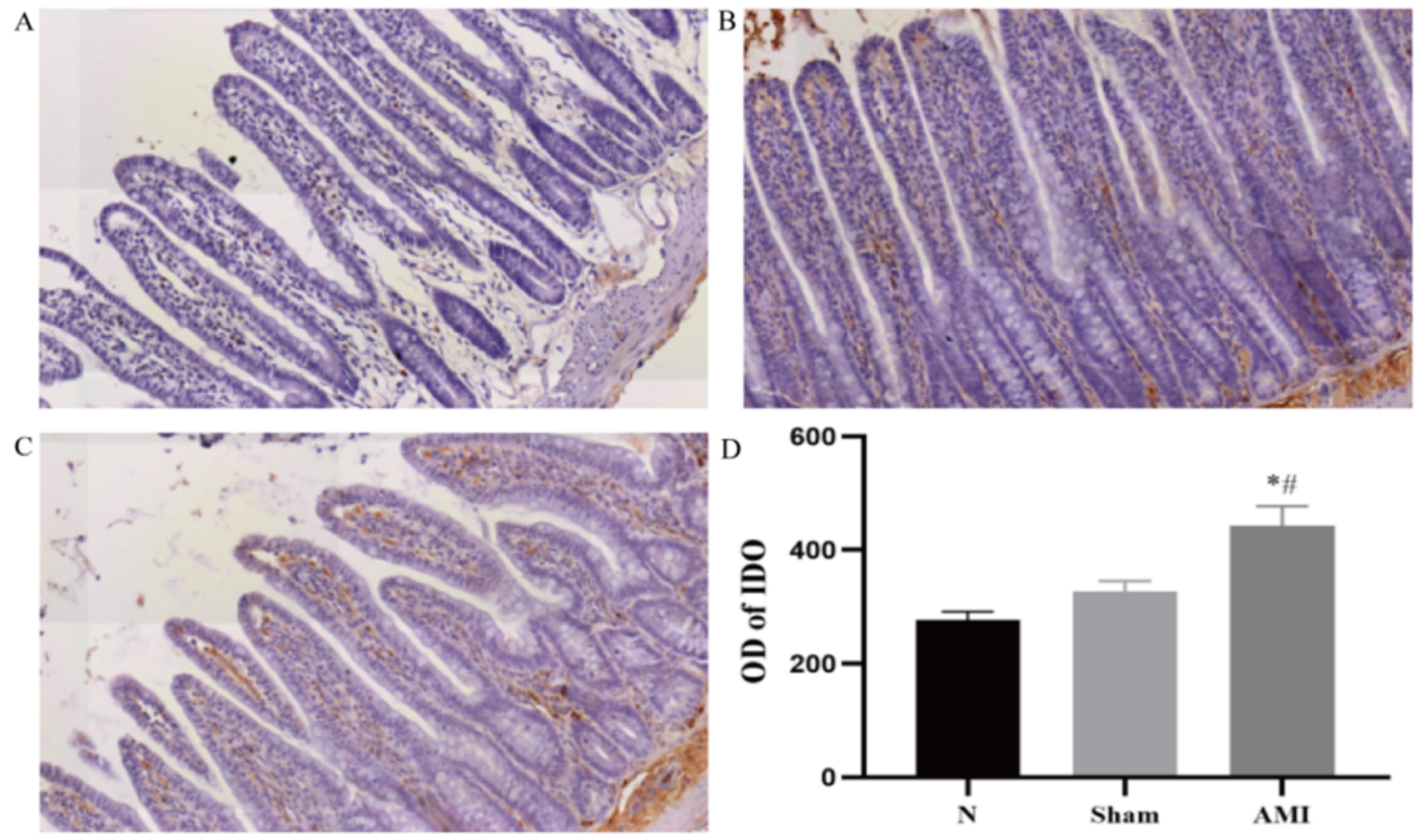

Figure 8

The expression of IDO.(40x). Arrows indicate IDO-positive cells. (A) N group. (B) Sham group. (C) AMI group. (D)The optical density of IDO from three groups (mean $\pm S E M, n=10) .{ }^{*}<0.01$ vs $N$ group, $\# P<0.01$ vs Sham group.

A

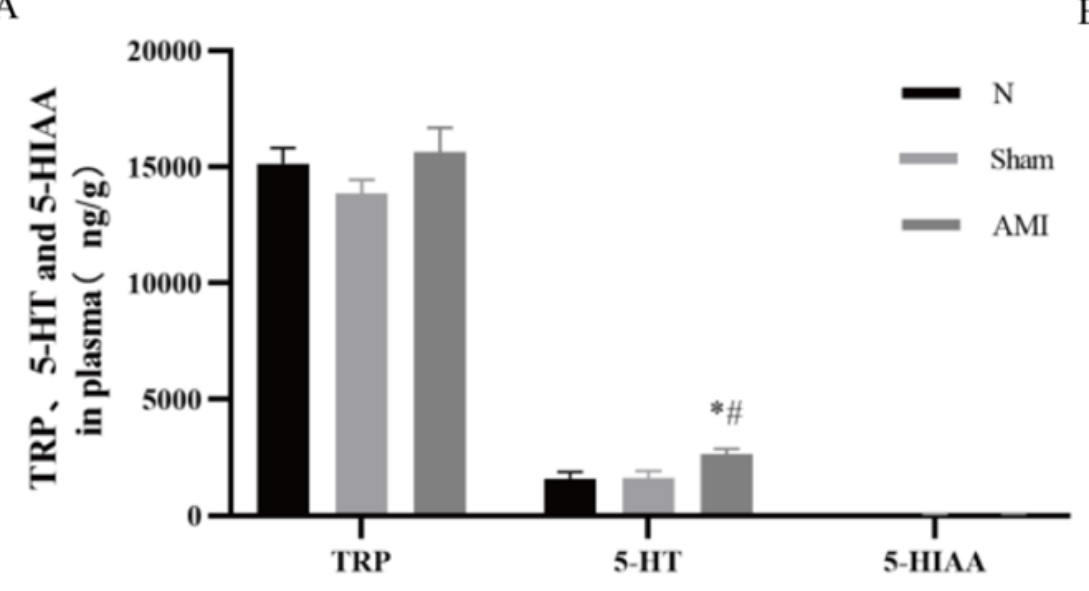

B

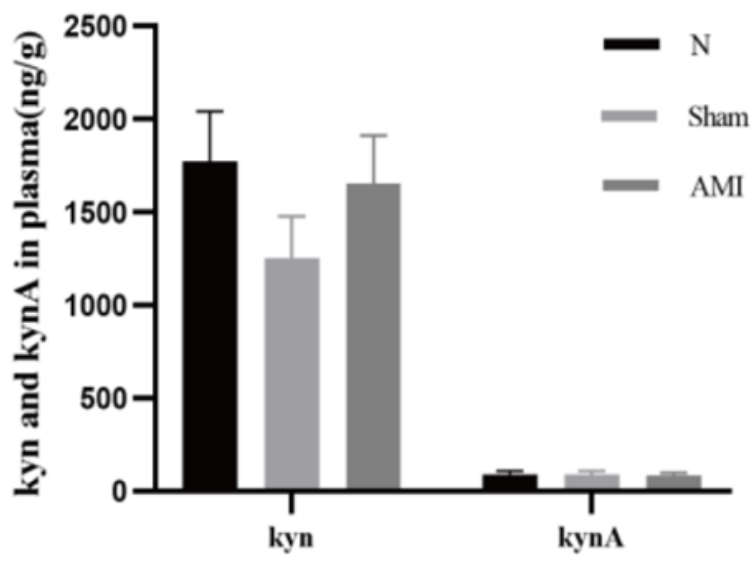

Figure 9

Tryptophan and its metabolites in the plasma(mean \pm SEM, $n=6$ ). (A)TRP, 5-HT and 5-HIAA. (B) Kyn and KynA. *Pख0.05 vs N group; \# Pख0.05 vs Sham group. 

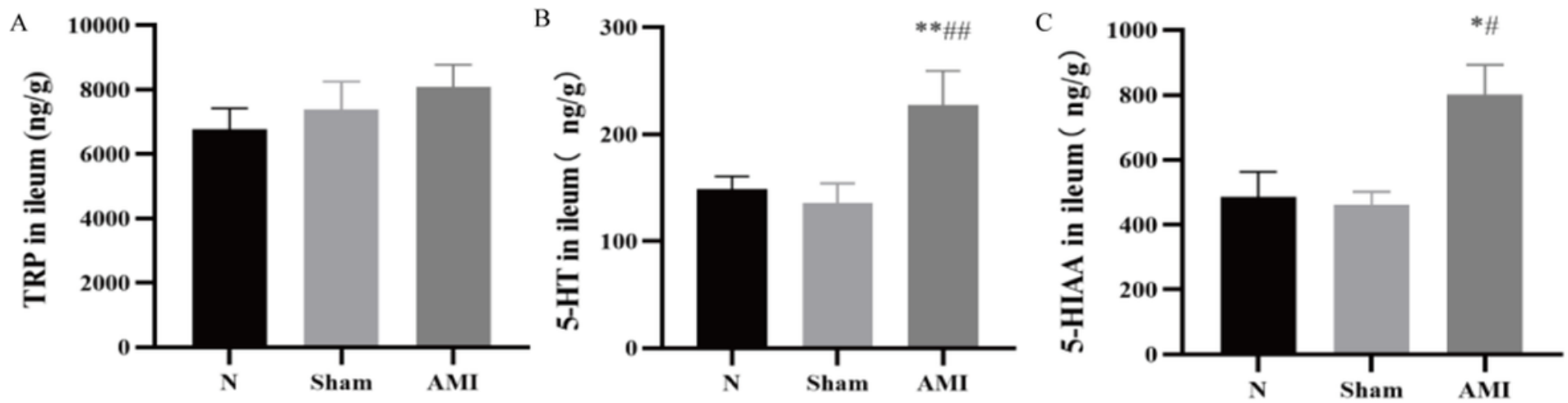

Figure 10

Tryptophan and its metabolites in the ileum (mean \pm SEM, $n=6$ ).(A)Tryptophan in the i-leum.(B) $5-H T$ in the

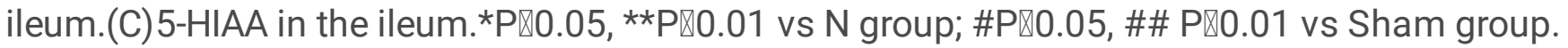

